\title{
JOINT DISCUSSION
}

Although this twisting process gives a satisfactory storage of energy, it does not immediately provide for the almost catastrophic discharge occurring in flares. For this, it appears necessary that two such filaments should have point contact. In the case where the longitudinal components of the magnetic field are in opposite directions, but where the rotary components are in the same sense, the penetration of the filaments at the point of contact annihilates the longitudinal components. This leaves a neutral point on the axis of the combined filaments at the contact point, and provides conditions favourable to the pinch effect. The electromagnetic forces then act in the sense of drawing the two bundles together, so that the contact point will run along the length of the filaments. A necessary condition is that the final stages of penetration occur quickly, in a time of a few seconds. It seems possible to satisfy this condition in the partially ionized gas of the lower chromosphere, provided the density is not greater than about ${ }^{10} 0^{12}$ atoms $/ \mathrm{cm}^{3}$.

This process can account for an explosive release of energy.

\section{BURSTS OF MICROWAVE RADIO EMISSION ASSOCIATED WITH SOLAR FLARES}

G. B. GELFREICH, V. N. IKHSANOVA, N. L. KAIDANOVSKY, N. S. SOBOLEVA, G. M. TIMOFEEVA AND N. V. UMETSKY

The 'burst' observations at Pulkovo have been made with a polarimeter using a 4-metre diameter reflector and the large Pulkovo radio telescope with a fan-beam diagram about $I^{\prime}$ in width. Nine bursts have been recorded during 250 hours of observations since July I957, seven of them being identified in time with solar flares.

The bursts are characterized by partial circular polarization, which ranges from 5 to $13 \%$. The sign of polarization corresponds to an extraordinary mode for the dominating magnetic field in the flare region.

The emitting regions seem to be situated in the corona at $0 \cdot I-0 \cdot 2 R_{\odot}$ above the photosphere. No fast movements of these regions were observed. At least on I958 March 3 the radial velocity of the burst was less than $150 \mathrm{~km} / \mathrm{sec}$. The areas of the bursts do not differ significantly from those of the flares. A brightness temperature of $1 \mathrm{ro}^{6}-\mathrm{IO}^{7}{ }^{\circ} \mathrm{K}$ is usual, but it can rise to $10^{8}{ }^{\circ} \mathrm{K}$ for some outstanding bursts.

A smooth curve of intensity is typical, with an abrupt growth and an exponential fall, the time constants of these exponents ranging from 0.3 to $15^{\mathrm{m}}$.

It is difficult to account for the observed phenomena in terms of plasma oscillations. If we assume that, in some small region of the corona, an abrupt heating takes place followed by diffusion of thermal energy, the observed intensity curve could be explained. The smooth run of the intensity curve and the sign of polarization (an extraordinary mode) are in agreement with the thermal mechanism assumption.

\section{r6. I.G.Y. SOLAR FLARE STUDIES}

\section{R. COATES, S. EDELSON, T. MCCULLOUGH AND N. SANTINI}

Since the beginning of the I.G.Y. the U.S. Naval Research Laboratory has been conducting a programme of simultaneous observations of solar activity in both $\mathrm{H} \alpha$ and radio wavelengths of 9.4 and $3.2 \mathrm{~cm}$. The analysis of the $\mathrm{H} \alpha$ and $9.4 \mathrm{~cm}$. observations for the first year of the I.G.Y. indicates that $99 \%$ of the radio bursts are coincident with $\mathrm{H} \alpha$ events, and that on the average the $9.4 \mathrm{~cm}$ bursts of types simple $\mathrm{I}$ and simple 2 start at the beginning of the $\mathrm{H} \alpha$ flare and reach a maximum midway between the $\mathrm{H} \alpha$ flare start and the flare maximum.

The very preliminary analysis of large complex $9.4 \mathrm{~cm}$ bursts indicates a very close relation between the radio intensity peaks and the brightenings of different eruptive centers of the same flare. 